\title{
Indução de resistência à intoxicação por Palicourea aeneofusca (Rubiaceae) mediante administração de doses sucessivas não tóxicas ${ }^{1}$
}

\author{
Murilo Duarte de Oliveira ${ }^{2}$, Franklin Riet-Correa ${ }^{3}$, Fabrício K.L. Carvalho ${ }^{3}$, \\ Genilson B. Silva ${ }^{2}$, Walkleber S. Pereira ${ }^{2}$ e Rosane M.T. Medeiros ${ }^{3 *}$
}

\begin{abstract}
Oliveira M.D., Riet-Correa F., Carvalho F.K.L., Silva G.B., Pereira W.S. \& Medeiros R. M.T. 2013. [Induction of resistance to Palicourea aeneofusca (Rubiaceae) poisoning by the continuous administration of non-toxic doses.] Indução de resistência à intoxicação por $P a$ licourea aeneofusca (Rubiaceae) mediante administração de doses sucessivas não tóxicas. Pesquisa Veterinária Brasileira 33(6):731-734. Hospital Veterinário, CSTR, Universidade Federal de Campina Grande, Patos, PB 58700-000, Brazil. E-mail: rmtmed@uol.com.br

Palicourea aeneofusca (Müll. Arg.) Standl. is a toxic plant which contains sodium monofluoroacetate (MFA). With the objective to investigate if repeated non-toxic doses of P. aeneofusca induce resistance to the intoxication by this plant, 12 goats were distributed in two similar groups. In Group 1, resistance was induced by the administration of the dry plant, during four alternate periods: $0.02 \mathrm{~g} / \mathrm{kg}$ during 5 days, $0.02 \mathrm{~g} / \mathrm{kg}$ during 5 days, $0.03 \mathrm{~g} / \mathrm{kg}$ during 5 days, and $0.03 \mathrm{~g} / \mathrm{kg}$ during 5 days. Between the first and second period of administration and between the second and the third period, the goats did not ingest P. aeneofusca for 10 days. Between the third and the fourth administration period the goats did not ingest the plant during 15 days. One goat died suddenly during the third administration period when was ingesting $0.03 \mathrm{~g} / \mathrm{kg}$. The goats from Group 2 were not adapted to the consumption of $P$. aeneofusca. Fifteen days after the end of the adaptation period in Group 1, both groups ingested dry P. aeneofusca in the daily dose of $0.03 \mathrm{~g} / \mathrm{kg}$ during 19 days. From day 20 the daily dose was increased to $0.04 \mathrm{~g} / \mathrm{kg}$, which was ingested for 12 days. The goats that showed clinical signs were removed from the experiment immediately after the observation of first signs. One goat from Group 2 showed clinical signs of poisoning and died on the $12^{\text {th }}$ day of ingestion, and two showed clinical signs on day $24^{\text {th }}$; one recovered and the other died. At the end of the 31 days administration period, a new group (Group 3) with three goats was introduced in the experiment to investigate if the goats that did not become poisoned in Group 2 had acquired resistance. The three goats from Group 1, five goats from Group 1, and three from Group 2 started to ingest a daily dose of $0.06 \mathrm{~g} /$ $\mathrm{kg}$ of dry P. aeneofusca. On the third day of ingestion the three goats from Group 3 showed clinical signs. Two died suddenly and another recovered 10 days after the end of ingestion. All goats of Groups 1 and 2 ingested $0.06 \mathrm{~g} / \mathrm{kg} /$ day during nine days without showing clinical signs. These results demonstrated that non-toxic repeated doses of P. aeneofusca increase significantly the resistance to the poisoning, and that this technique possibly could be used to control the poisoning by P. aeneofusca or other toxic Palicourea species. The results of previous research work suggest that resistance is due to the proliferation of MFA degrading bacteria in the rumen.

INDEX TERMS: Poisonous plants, acute cardiac insufficiency, sodium monofluoroacetate, Palicourea aeneofusca, Palicourea spp., resistance to plant poisoning, goats.
\end{abstract}

\footnotetext{
${ }^{1}$ Recebido em 30 de novembro de 2012.

Aceito para publicação em 27 de abril de 2013.

${ }^{2}$ Laboratório de Caprinocultura, Centro de Ciências Humanas, Sociais e Agrárias(CCHSA), Universidade Federal da Paraíba (UFPB), Bananeira, PB 58220-000, Brasil.

${ }^{3}$ Hospital Veterinário, Centro de Saúde e Tecnologia Rural (CSTR), Universidade Federal de Campina Grande (UFCG), Patos, PB 58700-000, Brasil. *Autor para correspondência: rmtmed@uol.com.br
}

RESUMO.- Com o objetivo de comprovar se doses não tóxicas repetidas de Palicourea aeneofusca (Müll. Arg.) Standl. criam resistência à intoxicação, 12 caprinos foram distribuídos aleatoriamente em dois grupos experimentais de seis animais cada. No Grupo 1 foi induzida resistência mediante a administração, durante quatro períodos alternados, de $0,02 \mathrm{~g} / \mathrm{kg}$ das folhas dessecadas de $P$. aeneofusca durante 
5 dias, $0,02 \mathrm{~g} / \mathrm{kg}$ durante 5 dias, $0,03 \mathrm{~g} / \mathrm{kg}$ durante 5 dias e $0,03 \mathrm{~g} / \mathrm{kg}$ por mais 5 dias. Entre o primeiro e o segundo período de administração e entre o segundo e o terceiro período os animais não receberam planta por 10 dias consecutivos e entre o terceiro e quarto período de administração os animais permaneceram 15 dias sem ingerir a planta. Um caprino morreu subitamente quando estava recebendo $0,03 \mathrm{~g} / \mathrm{kg}$ da planta, no terceiro período de administração. 0 Grupo 2 não foi adaptado ao consumo de $P$. aeneofusca. Quinze dias após a adaptação ao consumo de P. aeneofusca do Grupo 1, os dois grupos receberam P. aeneofusca na dose diária de $0,03 \mathrm{~g} / \mathrm{kg}$ durante 19 dias. A partir do $20^{\circ}$ dia de administração continuada a dose diária de $P$. aeneofusca foi aumentada para $0,04 \mathrm{~g} / \mathrm{kg}$. Esta dose foi administrada por mais 12 dias. Os animais que mostraram sinais clínicos foram retirados do experimento imediatamente após a observação dos primeiros sinais. Um caprino do Grupo 2 apresentou sinais clínicos de intoxicação e morreu no $12^{\circ}$ dia de administração e dois apresentaram sinais clínicos no 24 o dia; um se recuperou e outro morreu. Após finalizada esta fase do experimento e para comprovar se os caprinos que não tinham adoecido no Grupo 2 tinham também adquirido resistência, foi introduzido outro grupo com três caprinos. Esses três caprinos (Grupo 3), os cinco caprinos do Grupo 1 e os três sobreviventes do Grupo 2, ingeriram uma dose diária de $0,06 \mathrm{~g} / \mathrm{kg}$. Os três caprinos do Grupo 3 adoeceram no terceiro dia após o início da ingestão, dois morreram em forma hiperaguda e o outro recuperou-se após 10 dias. Todos os caprinos dos Grupos 1 e 2 ingeriram P. aeneofusca na dose de $0,06 \mathrm{~g} / \mathrm{kg} /$ dia durante nove dias sem apresentar nenhum sinal clínico. Os resultados deste trabalho demonstram que a administração de doses não tóxicas repetidas de $P$. aeneofusca aumentam significativamente á resistência à intoxicação e que esta técnica poderia ser utilizada para o controle da intoxicação por $P$. aeneofusca e outras espécies de Palicourea com similar toxicidade. Os resultados de pesquisas anteriormente realizados sugerem que a resistência à intoxicação por plantas que contêm MFA é devida a proliferação de bactérias que degradam MFA no rúmen.

TERMOS DE INDEXAÇÃO: Plantas tóxicas, insuficiência cardíaca aguda, monofluoroacetato de sódio, Palicourea aeneofusca, Palicourea spp., resistência à intoxicação por planta, caprinos.

\section{INTRODUÇÃO}

No Brasil existem pelo menos 13 plantas que causam insuficiência cardíaca aguda em ruminantes. 0 monofluoroacetato de sódio (MFA) foi determinado como o composto tóxico encontrado em Palicourea marcgravii (Oliveira 1963), Palicourea aeneofusca (Lee et al. 2012), Amorimia (Mascagnia) rigida (Cunha et al. 2012, Lee et al. 2012), Amorimia septentrionalis, Amorimia (Mascagnia) pubiflora (Lee et al. 2012) e Tanaecium bilabiatum (Arrabidaea bilabiata) (Krebs et al. 1994).

Na Paraíba são conhecidas duas plantas que contêm MFA e causam insuficiência cardiaca aguda, $A$. septentrionalis, anteriormente identificada como Amorimia (Mascagnia) rigida e $P$. aeneofusca (Vasconcelos et al. 2008).

Experimentalmente $P$. aeneofusca causou morte em ca- prinos (Passos 1983) e bovinos (Tokarnia \& Döbereiner 1982) nas doses de 0,6 e 0,75g/kg de peso corporal (g/ $\mathrm{kg}$ ), respectivamente. Os sinais clínicos são caracterizados por taquicardia, jugular ingurgitada com pulso venoso positivo, taquipnéia com respiração irregular ou abdominal, andar cambaleante, quedas e decúbito esternal evoluindo para decúbito lateral, seguidos de morte. Na necropsia geralmente não há lesões macroscópicas de significância. Na histologia a lesão mais frequente, observada nos rins em aproximadamente $50 \%$ dos animais intoxicados, é a severa vacuolização e picnose das células epiteliais dos túbulos contornados distais (Tokarnia \& Döbereiner 1982, Passos 1983).

Em trabalhos realizados pelo nosso grupo de pesquisa foi observado que animais que recebem diariamente doses não tóxicas de $A$. septentrionalis apresentam sinais clínicos, mas geralmente se recuperam se a administração é suspensa imediatamente após a observação dos primeiros sinais (Duarte et al. 2013). Após o consumo da planta é necessário um período para que o MFA seja detoxificado pelo organismo que de acordo com Tokarnia et al. (2012) varia entre oito a 14 dias para A. rigida e pode ser de dois a cinco dias para P. marcgravii.

Uma forma de controlar as intoxicações por plantas que contem MFA é o isolamento de bactérias que hidrolisam MFA de forma a serem introduzidas no rúmen de animais susceptíveis para lhes conferir resistência. Dentro dessa linha de pesquisa foram isoladas, na Paraíba, bactérias que hidrolisam MFA do solo, de plantas e do rúmen de caprinos que nunca tinham ingerido plantas que contêm MFA (Camboim et al. 2012a,b). Foi demonstrado, também, que bactérias que hidrolisam MFA são muito mais frequentes do que se pensava anteriormente (Camboim 2012) e que muito provavelmente a administração de plantas que contem MFA induz a multiplicação dessas bactérias que ocorrem normalmente no rúmen, induzindo resistência à intoxicação. Foi demonstrado que a administração diária e contínua de doses não tóxicas de $A$. septentrionalis administradas em períodos alternados, que permitem a detoxificação do MFA, induz resistência contra a intoxicação por esta planta e que essa resistência pode ser transmitida de um animal resistente para um animal susceptível mediante a transfaunação de conteúdo ruminal (Duarte et al. 2013).

0 presente trabalho teve como objetivo determinar se é possível induzir resistência à intoxicação por $P$. aeneofusca mediante a administração de doses diárias não tóxicas da mesma por períodos alternados.

\section{MATERIAL E MÉTODOS}

Para a realização do experimento, folhas de Palicourea aeneofusca foram coletadas no Município de Areia, Paraíba, Brasil $\left(07^{0} 04^{\prime} 02^{\prime \prime} \mathrm{S}, 37^{0} 16^{\prime} 51^{\prime \prime} \mathrm{W}\right.$ e altitude de 560 metros acima do nível do mar) e desidratadas em temperatura ambiente por quatro dias. Após secas foram trituradas em moinho e guardadas em potes fechados. Amostras da planta foram enviadas Poisonous Plant Research Laboratory, em Logan, Estados Unidos, para determinação da concentração de MFA, detectando-se que a mesma continha $0,09 \pm 0,05 \%$ de MFA (Lee et al. 2012). 
Quadro 1. Indução de resistência à ingestão de Palicourea aeneofusca administrando planta seca em doses
diárias repetidas por períodos variáveis (Grupo 1) e desafio mediante administração continuada da planta (Grupos 1, 2 e 3)

\begin{tabular}{|c|c|c|c|c|c|c|c|c|c|c|c|}
\hline \multirow[t]{2}{*}{ Grupo } & \multicolumn{11}{|c|}{ Doses e períodos de ingestão, períodos sem ingestão e caprinos que adoeceram sobre o total em cada período } \\
\hline & \multicolumn{8}{|c|}{ Período de indução de resistência } & \multicolumn{3}{|c|}{ Período de desafio } \\
\hline \multirow[t]{2}{*}{ G1 } & $0,02 \mathrm{~g} / \mathrm{kg}$ & PSI* & $0,02 \mathrm{~g} / \mathrm{kg}$ & PSI & $0,03 \mathrm{~g} / \mathrm{kg}$ & PSI & $0,03 \mathrm{~g} / \mathrm{kg}$ & PSI & $0,03 \mathrm{~g} / \mathrm{kg}$ & $0,04 \mathrm{~g} / \mathrm{kg}$ & $0,05 \mathrm{~g} / \mathrm{kg}$ \\
\hline & 5 dias & 10 dias & 5 dias & 10 dias & 5 dias & 15 dias & 5 dias & 15 dias & 19 dias & 12 dias & 9 Dias \\
\hline Doentes/total & $0 / 6$ & $0 / 6$ & $0 / 6$ & $0 / 6$ & $1 / 6$ & $0 / 5$ & $0 / 5$ & $0 / 5$ & $0 / 5$ & $0 / 5$ & $0 / 5$ \\
\hline \multirow[t]{2}{*}{ G2 } & \multirow{2}{*}{\multicolumn{8}{|c|}{ Seis caprinos que não foram adaptados }} & $0,03 \mathrm{~g} / \mathrm{kg}$ & $0,04 \mathrm{~g} / \mathrm{kg}$ & $0,05 \mathrm{~g} / \mathrm{kg}$ \\
\hline & & & & & & & & & & & 9 dias \\
\hline Doentes/total & & & & & & & & & $1 / 6$ & $2 / 5$ & $0 / 3$ \\
\hline G3 & \multicolumn{10}{|c|}{ Três caprinos que não foram adaptados } & $\begin{array}{c}0,05 \mathrm{~g} / \mathrm{kg} \\
9 \mathrm{dias}\end{array}$ \\
\hline Doentes/total & & & & & & & & & & & $3 / 3$ \\
\hline
\end{tabular}

* PSI = Sem ingestão.

O Experimento foi realizado na cidade de Bananeiras, Paraíba, no Laboratório de Caprinocultura do Centro de Ciências Humanas, Sociais e Agrárias (CCHSA) da Universidade Federal da Paraíba (UFPB). Inicialmente foram utilizados 12 caprinos, hígidos, das raças Alpino e Saanen pesando entre 15 e $25 \mathrm{~kg}$, de ambos os sexos e distribuídos aleatoriamente em dois grupos experimentais de seis animais cada. Com a finalidade de verificar se é possível induzir resistência, os animais do Grupo 1 (Caprinos 1-6) receberam, mediante a administração diária, em períodos alternados, $0,02 \mathrm{~g} / \mathrm{kg}$ de $P$. aeneofusca dessecada durante 5 dias, $0,02 \mathrm{~g} / \mathrm{kg} \mathrm{du}-$ rante 5 dias, $0,03 \mathrm{~g} / \mathrm{kg}$ durante 5 dias e $0,03 \mathrm{~g} / \mathrm{kg}$ por mais 5 dias. Entre o primeiro e o segundo período de administração e entre o segundo e o terceiro período os animais não receberam planta por 10 dias consecutivos e entre o terceiro e quarto período de administração os animais permaneceram 15 dias sem ingerir a planta (Quadro 1). Durante cada período de fornecimento da planta foram realizados exames clínicos que consistiam em verificação das frequências cardíaca e respiratória, da temperatura retal e do comportamento do animal.

O Grupo 2 (Caprinos 7-12) não foi adaptado ao consumo de $P$. aeneofusca. Quinze dias após o final da adaptação ao consumo de $P$. aeneofusca do Grupo 1 , os dois grupos receberam $P$. aeneofusca na dose diária de $0,03 \mathrm{~g} / \mathrm{kg}$ durante 19 dias. A partir do $20^{\circ}$ dia de administração continuada a dose diária de P. aeneofusca foi aumentada para $0.04 \mathrm{~g} / \mathrm{kg}$. Esta dose foi administrada por mais 12 dias.

Considerando que após 31 dias de administração da planta em forma continuada, em doses crescentes, havia 5 caprinos do Grupo 1 e três do Grupo 2 que não adoeceram, foi introduzido um novo grupo de três caprinos (Caprinos 13-15) para testar se os oito animais mencionados anteriormente apresentavam resistência superior à do início do experimento. A partir do dia 32 os cinco caprinos do Grupo 1, os três do Grupo 2 e os três do Grupo 3 passaram a receber diariamente $0,06 \mathrm{~g} / \mathrm{kg}$ de $P$. aeneofusca seca, até o dia 40.

Durante todo o experimento, nos animais que apresentavam sinais clínicos foi suspenso o fornecimento da planta no mesmo dia que adoeceram, sendo retirados do experimento e colocados em gaiolas afastadas em local tranquilo.

Os animais que morreram foram necropsiados. Amostras de órgãos das cavidades, abdominal, torácica e sistema nervoso central foram coletadas, fixadas em formol a $10 \%$, incluídas em parafina, cortadas a 5-6 $\mu \mathrm{m}$ e coradas pela hematoxilina-eosina para exame histológico. Para a interpretação das lesões microscópicas cardíacas foram coletadas, no Abatedouro Municipal de Patos, amostras do coração de 10 caprinos normais, que foram processadas para estudo histológico em forma semelhante à dos caprinos intoxicados.

\section{RESULTADOS}

No Grupo 1, um animal (Caprino 1) morreu, sem que fossem observados sinais clínicos, durante o período de indução de resistência, no dia 34, 4 dias após o início da ingestão da dose diária de $0,03 \mathrm{~g} / \mathrm{kg}$. Em consequência disso o intervalo entre as administrações, previsto inicialmente para 10 dias, foi aumentado para 15 dias. Após o período de indução de resistência nenhum animal do Grupo 1 apresentou sinais clínicos durante os 40 dias da fase de desafio mediante fornecimento de Palicourea aeneofusca em doses crescentes (Quadro 1).

No Grupo 2, um animal (Caprino 12) apresentou sinais clínicos e morreu no $12^{\circ}$ de ingestão da planta. No $24^{\circ}$ dia adoeceram outros dois caprinos (Caprinos 8 e 10) deste grupo. 0 Caprino 10 se recuperou em 6 dias e o Caprino 8 estava se recuperando 48 horas após o final da ingestão, quando foi solto numa pastagem e no retorno da mesma morreu após apresentar queda e movimentos de pedalagem. Nenhum outro animal do Grupo 2 adoeceu até o 40o dia (Quadro 1).

Dos animais do Grupo 3, todos adoeceram três dias após o início da ingestão de $0,06 \mathrm{~g} / \mathrm{kg}$, enquanto que os três animais sobreviventes do Grupo 2 o os 5 animais do Grupo 1 receberam essa dose durante 9 dias sem apresentar sinais. Dos três animais do Grupo 3 que adoeceram os Caprinos 14 e 15 morreram em forma hiperaguda e o Caprino 13 recuperou-se após 10 dias. As doses administradas a cada grupo, os períodos de administração, os períodos nos que a planta não foi administrada e a observação de animais doentes apresentam-se no Quadro 1.

Os Caprinos 8, 12, 14 e 15 que adoeceram e morreram apresentaram clinicamente apatia, anorexia, taquicardia, ligeira perda de equilíbrio, muitas vezes com andar cambaleante e aumento progressivo da dificuldade locomotora e elevado grau de dificuldade quando iam deitar-se. A micção era frequente. Na fase agônica da doença todos os animais caiam em decúbito lateral, esticavam os membros, faziam movimentos de pedalagem e apresentavam opistótono, nistagmo e mugidos intensos. A morte ocorria de 10 a 20 minutos após o início dos sinais clínicos.

Nos Caprinos 10 e 13, que se recuperaram, os sinais eram mais brandos observando-se apatia e anorexia, permanecendo em decúbito esternal por longos períodos. A 
recuperação ocorreu em três dias no Caprino 10 e em 10 dias no Caprino 13. Neste último registrou-se uma perda de peso de $6 \mathrm{~kg}$.

Não foram observadas lesões macroscópicas nos cinco caprinos que morreram durante o experimento. No estudo histológico, os rins dos Caprinos 1 e 14 apresentaram vacuolização e picnose de células epiteliais de raros túbulos contornados distais. No Caprino 8, além de vacuolizados, numerosos túbulos apresentava necrose e desprendimento de células epiteliais. No Caprino 1 não foram observadas lesões cardíacas. No coração do Caprino 12 havia fibras musculares eosinofílicas e com picnose nuclear e numerosas fibras de Purkinje apresentavam-se vacuolizadas. Nos Caprinos 8, 13 e 14 havia discreta vacuolização das fibras de Purkinje, mais marcada nas fibras localizadas no miocárdio do que das localizadas no endocárdio. Estas lesões não foram observadas no coração de 10 caprinos coletados no abatedouro de Patos e utilizadas como controle.

\section{DISCUSSÃO}

Os resultados deste trabalho demonstram que a administração de doses não tóxicas repetidas de Palicourea aeneofusca aumentam significativamente á resistência à intoxicação. Resultados semelhantes foram obtidos por Duarte et al. (2013) com a administração de Amorimia septentrionalis a caprinos. 0 fato de três animais do Grupo 2 não terem apresentados sinais clínicos após ingerir a planta durante 12 dias na dose diária de $0,03 \mathrm{~g} / \mathrm{kg}$ e outros 20 dias na dose de $0,04 \mathrm{~g} / \mathrm{kg}$ deveu-se, provavelmente, a que adquiriram resistência durante o período de administração da planta. Essa hipótese foi comprovada na segunda fase do experimento quando foi introduzido outro grupo de três animais no experimento. Enquanto esses três animais introduzidos recentemente no experimento adoeceram após três dias de ingestão da dose de 0,06g/ kg, os animais dos Grupos 1 e 2 não apresentaram nenhum sinal de intoxicação após ingerirem diariamente a mesma dose durante nove dias.

Por outro lado, o fato de que essa resistência pode ser transmitida de animais resistentes para animais susceptíveis mediante a transfaunação de conteúdo ruminal (Duarte et al. 2013) comprova que a resistência deve-se à proliferação de bactérias que hidrolisam o MFA. A degradação microbiológica de MFA é catalisada por fluoroacetato dehalogenases que clivam a forte ligação do carbono-fluor (Fetzner \& Lingens 1994). Os resultados obtidos neste experimento e as observações de Duarte et al. (2013) com $A$. septentrionalis, mostram que há numerosas bactérias que degradam MFA (Camboim 2012). A repetida administração das plantas que contem MFA, em doses pequenas, favoreceria a multiplicação dessas bactérias, que normalmente ocorrem no rúmen dos animais.

No seu conjunto, os resultados desta experimentação com P. aeneofusca e dos trabalhos de Duarte et al. (2013), Camboin (2012) e Camboin et al. $(2011,2012)$ sugerem que há três possibilidades a serem exploradas para o con- trole de intoxicações por plantas que contêm MFA mediante o aumento da resistência dos animais: 1) a administração repetida, por períodos alternados, de doses não tóxicas da planta; 2) a administração de MFA em doses não tóxicas o que permitiria, também, a proliferação de bactérias que tenham atividade de dehalogenases; e 3) a administração de outro substrato, não tóxico, que estimule a proliferação de bactérias com atividade de dehalogenases. Dessas três possibilidades, a terceira pareceria a mais adequada, já que resultaria difícil, sem conhecer a concentração de MFA, administrar a planta com segurança a doses não tóxicas e também é difícil trabalhar com uma substância de alta toxicidade como o MFA.

\section{REFERÊNCIAS}

Cunha L.C., Pipole F., Carvalho L.R., Lago J.H.G. \& Görniak S.L. 2012. Isolation and characterization of sodium 2-fluoroacetate from Mascagnia rigida using chromatography and infrared spectroscopy. Toxicon 60:329-332.

Camboim E.K.A. 2012. Bactérias que degradam fluoroacetato. Tese de Doutorado, Programa de Pós-Graduação em Medicina Veterinária, Universidade Federal de Campina Grande, Patos, Paraíba. 81p.

Camboim E.K.A., Tadra-Sfeir M.Z., Souza E.M., Pedrosa F.O., Andrade P. P., McSweeney C.S., Riet-Corea F. \& Melo M.A. 2012a. Defluorination of sodium fluoroacetate by bacteria from soil and plants in Brazil. Scientific World Journal. Article ID 149893, 5 pages, Disponível em <http://www.tswj.com/2012/149893/> Acesso em 20 ago. 2012. doi:10.1100/2012/149893

Camboim E.K.A., Almeida A.P., Tadra-Sfeir M.Z., Junior F.G., Andrade P.P., McSweeney C.S., Riet-Correa F. \& Melo M.A. 2012b. Isolation of sodium fluoroacetate degrading bacteria from caprine rumen in Brazil. Scientific World Journal. Article ID 178254, 6 pages. Disponível em <http://www.tswj.com/2012/178254/> Acesso em 20 ago. 2012. doi:10.1100/2012/178254.10

Duarte A.L.L., Medeiros R.M.T., Carvalho F.K.L., Lee S.T., Cook D., Pfister J.A., Costa V.M.M. \& Riet-Correa F. 2013. Induction and transfer of resistance to poisoning by Amorimia (Mascagnia) septentrionalis in goats. J. Appl. Toxicol. (In publication). DOI 10.1002/jat.2860.

Fetzner S. \& Lingers F. 1994. Bacterial dehalogenases: biochemistry, genetics, and biotechnological applications. Microbiol. Rev. 58(4):641-685.

Krebs H.C., Kemmerling W. \& Habermehl G. 1994. Qualitative and quantitative determination of fluoroacetic acid in Arrabidea bilabiata and Palicourea marcgravii by F-NMR spectroscopy. Toxicon 32:909-913.

Lee S.T., Cook D., Riet-Correa F., Pfister J.A., Anderson W.R., Lima F.G. \& Gardner D. 2012. Detection of monofluoracetate in Palicourea and Amorimia species. Toxicon 60:791-796.

Oliveira M.M. 1963. Chromatographic isolation of monofluoroacetic acid from Palicourea marcgravii St. Hil. Experientia 19(11):586-587.

Passos D.A. 1983. Intoxicação Experimental em Caprinos (Capra hircus) por Palicourea aeneofusca (M. Arg.) Standl. (Rubiaceae). Tese de Mestrado, Universidade Federal Rural de Pernambuco, Recife, PE. 40p.

Tokarnia C.H., Döbereiner J., Couceiro J.E.M. \& Cordeiro Silva A.C. 1982. Intoxicação por Palicourea aeneofusca (Rubiaceae): a causa de mortes súbitas em bovinos na Zona da Mata Pernambucana. Pesq. Vet. Bras. 3(3):75-79.

Tokarnia C.H., Brito M.F., Barbosa J.D., Peixoto P.V. \& Döbereiner J. 2012. Plantas tóxicas do Brasil. Editora Helianthus, Rio de Janeiro. 586p.

Vasconcelos J.S., Riet-Correa F., Dantas A.F.M., Medeiros R.M.T. \& Dantas A.J.A. 2008. Mortes súbitas em bovinos causadas por Palicourea aeneofusca (Rubiaceae) e Mascagnia rigida (Malpighiaceae) na Zona da Mata Paraibana. Pesq. Vet. Bras. 28(10):457-460. 